\title{
Du flou des images astronomiques à un prix Nobel de physique
}

Pierre Léna ${ }^{(1,2)}$ (pierre.lena@obspm.fr) et Guy Perrin ${ }^{(1)}$ (guy.perrin@obspm.fr) (1) LESIA (Observatoire de Paris, Université PSL CNRS, Sorbonne Université, Université de Paris). Observatoire de Paris, 5 place Jules Janssen, 92195 Meudon Cedex (2) Université de Paris

Deux des lauréats du prix Nobel de physique 2020 sont primés pour leurs observations [1], qui ont confirmé l'existence d'un trou noir supermassif au centre de notre Galaxie.

En améliorant considérablement, jusqu'à un facteur de plus de cent-mille parfois, la résolution spatiale des télescopes utilisés, ils ont exploré un véritable laboratoire de relativité générale, au plus près de cette singularité de l'espace-temps.

Ces gains en résolution résultent d'un franchissement de la limitation imposée par l'atmosphère terrestre, grâce à l'interférométrie des tavelures (1970), puis l'optique adaptative (1989), enfin la mise en service de télescopes optiques géants (ca. 2000), et du réseau interférométrique du Very Large Telescope européen.

Cet article prolonge celui de $\mathrm{N}$. Deruelle et J.P. Lasota, "Le prix Nobel de physique 2020 , ou la consécration des trous noirs", Reflets de la physique 68 (2021) 20-23.

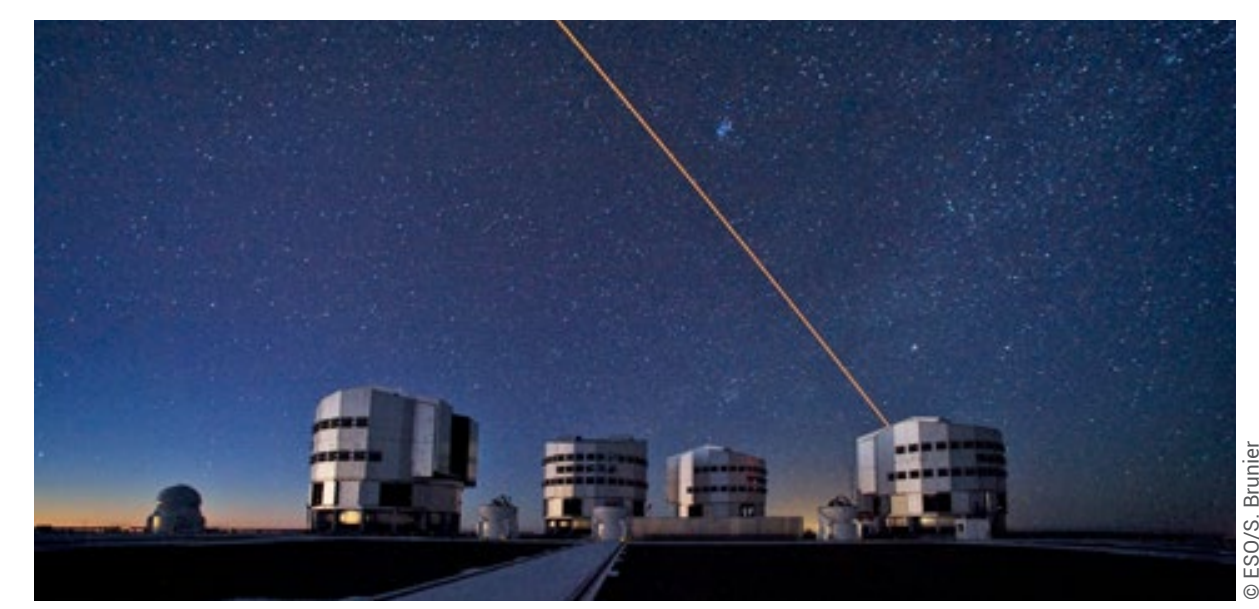

Le Very Large Telescope (VLT) de l'ESO durant les observations. Un laser est lancé du télescope UT4 pour créer une étoile artificielle à une distance de $90 \mathrm{~km}$ de la Terre. Ce laser fait partie du système d'optique adaptative qui permet aux astronomes d'éviter les effets de turbulence de l'atmosphère, et produit des images presque aussi nettes que si le télescope était dans l'espace (voir encadré, p. 23).

Galilée fut le premier, avec sa lunette, à dépasser le pouvoir de résolution angulaire de l'œil nu, et à mettre en évidence des détails inconnus sur des objets célestes. Depuis, cette quête du détail n'a jamais cessé. Malgré un diamètre $\mathrm{D}$ croissant (télescope Hale de $\mathrm{D}=5 \mathrm{~m}$, Mont Palomar, 1952), la résolution spatiale des télescopes optiques - lumière visible, puis infrarouge depuis quelques décennies demeurait limitée par la turbulence de l'atmosphère terrestre à environ une seconde d'arc (le seeing), et ne pouvait atteindre la résolution ultime $(\lambda / D)$ fixée par la diffraction de la lumière. L'accès à l'espace (Hubble, D = 2,4 m, 1989) s'en affranchissait, mais ce ne sera qu'en 2022 que le James Webb Space Telescope $(\mathrm{D}=6,4 \mathrm{~m})$ progressera dans cette voie.

Dès 1868, Hippolyte Fizeau avait proposé de mesurer le diamètre des étoiles en atteignant, ou même augmentant, la résolution $\lambda / \mathrm{D}$ par une méthode interférométrique. Albert Michelson mit en œuvre cette idée sur le télescope Hooker $(\mathrm{D}=2,5 \mathrm{~m}$, étendu ainsi à $6 \mathrm{~m}$ ) au Mont Wilson (Californie). En 1920, Francis Pease et lui furent les premiers à mesurer le diamètre angulaire d'une étoile, Bételgeuse (environ 40 millisecondes d'arc), améliorant d'un facteur vingt-cinq la limite imposée par le seeing. La physique de l'équilibre interne des étoiles, disposant ainsi de vérifications par l'observation, progressa alors rapidement.

Dans les années 1950, la radioastronomie aux longueurs d'onde supérieures au centimètre (moins affectées par l'atmosphère) exploita l'idée de Fizeau. La combinaison cohérente des rayonnements reçus par plusieurs télescopes distants, formant un interféromètre, synthétisa l'image que donnerait un télescope de plusieurs centaines de mètres de diamètre, voire davantage, dans le cas d'instruments situés à des distances intercontinentales. 
$>>$

Aux longueurs d'onde optiques, les effets de seeing et la précision instrumentale requise dissuadèrent longtemps un effort semblable, jusqu'en 1975 quand Antoine Labeyrie sut coupler de façon cohérente deux petits télescopes distants de 12 mètres, puis davantage, et mesurer le diamètre de l'étoile Véga avec une précision exceptionnelle. Ce résultat, et les entreprises parallèles qui suivirent dans le monde, influèrent de façon décisive sur la conception, alors en cours, duVery Large Telescope européen.

Quant à la limitation imposée aux télescopes optiques par le seeing, elle fut franchie progressivement à partir de 1970 par un traitement des images perturbées, proposé par Labeyrie, puis définitivement en 1989 grâce à l'optique adaptative (voir encadré, p. 23). Cette méthode de correction du front d'onde après sa traversée de l'atmosphère, en temps réel, restitua aux télescopes géants de la fin du $\mathrm{XX}^{\mathrm{e}}$ siècle la résolution limitée par la diffraction.

\section{Turbulence atmosphérique, limite de diffraction et interférométrie des tavelures}

En 1970, utilisant le télescope de $5 \mathrm{~m}$ du Mont Palomar, A. Labeyrie montre que la tache de seeing contient encore de l'information à haute fréquence spatiale sur la structure de l'objet [2]. Une transformation de Fourier permet de retrouver le module des composantes de hautes fréquences et donc, sans toutefois reconstituer une véritable image, d'obtenir une précieuse information sur les détails compris entre l'étalement du seeing et la limite $(\lambda / D)$ imposée par la diffraction. Cette démonstration a d'importantes conséquences. Dans le visible et le proche infrarouge, elle permet des mesures du diamètre de certaines étoiles brillantes, des condensations de formation d'étoiles, des éjections de matière par ces dernières. L'interférométrie des tavelures, comme elle est alors nommée, confronte les modèles de propagation lumineuse dans un milieu d'indice aléatoire - ici l'atmosphère - avec les observations. Sont ainsi mis en évidence : la dépendance chromatique de l'aire de cohérence du front d'onde, du temps de cohérence dans l'image, du domaine isoplanétique autour d'un point de celle-ci, ainsi que d'autres facteurs caractéristiques de la turbulence en un site donné, telle l'échelle externe ou la répartition verticale des couches turbulentes entre le sol et la tropopause. Par comparaison à la lumière visible, ces facteurs sont tous plus favorables dans le proche infrarouge, comme le montra François Roddier.

En 1977, s'inspirant de techniques numériques mises au point pour le traitement des images en radioastronomie (algorithme Clean), Gerd Weigelt montre qu'un traitement analogue (triple masking) permet à l'interférométrie des tavelures de récupérer l'intégralité d'une image jusqu'à la limite de diffraction, au prix d'une perte significative de rapport signal sur bruit. D'autres méthodes statistiques voient le jour, en particulier celles qui consistent à sélectionner, parmi les images aléatoires instantanées - poses de quelques centièmes de seconde figeant la turbulence -, les plus riches en hautes fréquences et à reconstruire une image par translation et addition de ces instantanés (lucky imaging, shift-and-add...).

Un pas essentiel est franchi avec la première démonstration de l'optique adaptative en astronomie, qui fut accomplie par une équipe française en 1989 et fournit une image à la limite de diffraction d'un télescope de 1,52 m [3]. Cet outil équipera naturellement le Very Large Telescope (VLT) de l'Observatoire européen austral (ESO), alors en construction sur le Cerro Paranal à $2630 \mathrm{~m}$ d'altitude dans le désert d'Atacama (Chili). Cet instrument géant entre en service dix ans plus tard, avec une optique adaptative dotée d'une caméra infrarouge (Naos-Conica), située au foyer de l'un des quatre télescopes de 8,2 m. Il en est de même au Keck Telescope au Mauna Kea (Hawai). Au Chili comme à Hawaï, la limite de diffraction à $\lambda=2,2 \mu \mathrm{m}$ est atteinte.

\section{Un trou noir potentiel au centre de la Voie lactée}

Entre 1974 et 1982, une source radio est identifiée au centre cinématique de notre Galaxie, situé dans la direction de la constellation du Sagittaire. Son nom, Sgr A*, en dérive. Dès 1985, à Berkeley, Charles Townes (prix Nobel de physique 1964 pour la conception du maser), avec notamment le jeune Reinhard Genzel, publie une étude sur cette région qui, à partir d'un ensemble d'observations radio et infrarouge, conclut à la présence probable d'un trou noir massif. Peu auparavant, l'existence de tels objets avait été postulée pour expliquer l'extrême intensité d'émission lumineuse par les quasars. Ce n'est qu'en 1998 que l'étude radioastronomique du mouvement de masers $\mathrm{H}_{2} \mathrm{O}$ autour de Sgr $A^{\star}$ donnera une première indication de la masse de cet objet. R. Genzel et A. Eckart installent une caméra infrarouge ( $32 \times 32$ pixels, format maximal à l'époque) sur le New Technology Telescope (3,58 m) de l'ESO au Chili. Le domaine infrarouge s'impose, vers 2,2 $\mu \mathrm{m}$, puisque ce n'est qu'à ces longueurs d'onde que les nuages denses de poussière, situés dans l'épaisseur du plan galactique, deviennent transparents. Appliquant la technique de sélection d'images ci-dessus, ils obtiennent une carte du champ d'étoiles entourant Sgr A* avec une résolution proche de 0,1 seconde d'arc. Ces observations sont poursuivies de façon continue jusqu'en 2002 et permettent de reconstruire les mouvements propres de plusieurs dizaines d'étoiles.

Utilisant la même technique de sélection d'images sur l'un des télescopes Keck de $10 \mathrm{~m}$ mis en œuvre à Hawaï à partir de 1998, Andrea Ghez à Berkeley publie un catalogue de ces étoiles, qui met également en évidence leurs trajectoires elliptiques [4]. Ces deux équipes concurrentes, mais qui échangent leurs résultats, déduisent par simple application de la troisième loi de Kepler une masse entre 2 et $810^{6} \mathrm{M}_{\text {Soleil }}$ pour l'objet central, dont la nature de trou noir devient de plus en plus probable. Mais ces mesures sont encore très loin de l'échelle spatiale à atteindre pour montrer que cette masse est circonscrite à l'horizon du trou noir. Il s'en faut d'un facteur 50000 en résolution!

Une étroite collaboration s'installe entre les équipes allemande et française, ces dernières ayant construit l'instrument Naos. Parmi toutes les étoiles environnant Sgr A^, l'une d'entre elles, S2, retient particulièrement l'attention avec une orbite fortement elliptique et une période de 16 ans, les passages au péricentre devant se produire en 2002 et 2018. S2 approche alors Sgr A* à une distance de 17 heureslumière, soit un angle de 12 millisecondes d'arc vu de la Terre. La détermination précise de l'orbite, en mesurant la vitesse projetée, bénéficie de la résolution accrue. Ceci permet d'affiner la détermination de la masse centrale et d'affirmer progressivement le caractère extrêmement compact 
Recevant d'une source ponctuelle une onde lumineuse plane monochromatique de longueur d'onde $\lambda$, l'objectif (de diamètre $D$ ) d'un télescope placé dans l'espace en donne en son foyer une image dont la distribution d'intensité est fixée par la figure d'Airy (une tache brillante entourée de cercles concentriques de plus faible luminosité), étalée par la diffraction et d'une largeur angulaire à mi-hauteur d'environ $\lambda / D$. Mais si l'onde a traversé l'atmosphère terrestre où les inhomogénéités aléatoires de température créent des inhomogénéités d'indice de réfraction, la surface d'onde abordant l'objectif est comme « cabossée en phase ". Ses retards ou avances de phase, rapidement variables dans le temps et aléatoires sur la surface de l'onde, peuvent être de l'ordre de la longueur d'onde, voire supérieurs. Très élevés et affectant l'amplitude, ils sont responsables de la scintillation des étoiles. Plus faibles, ils provoquent un étalement de l'image focale, déjà compris par Newton et appelé seeing par les astronomes. Cette tache de seeing a généralement une dimension de l'ordre de la seconde d'arc, soit près de vingt fois la limite de diffraction à $\lambda=2 \mu \mathrm{m}$ pour un télescope de $8 \mathrm{~m}$ : la perte de résolution angulaire est dramatique.

L'optique adaptative est une méthode active de « décabossage » du front d'onde, qui restitue sa phase d'origine et donc la qualité ultime de l'image [11]. Pour atteindre ce but, trois éléments sont utilisés (fig. E1). Prélevant une partie de l'énergie du front d'onde, l'analyseur de front d'onde l'échantillonne et en mesure rapidement (à l'échelle du kHz) les écarts de phase. La déformation de la surface d'un petit miroir, placé sur une pupille image de l'objectif, est obtenue par une grille d'actionneurs, qui sont commandés par un calculateur à partir des écarts mesurés. La méthode connait toutefois une limite, Iorsque l'objet étudié est trop peu brillant pour que l'analyseur

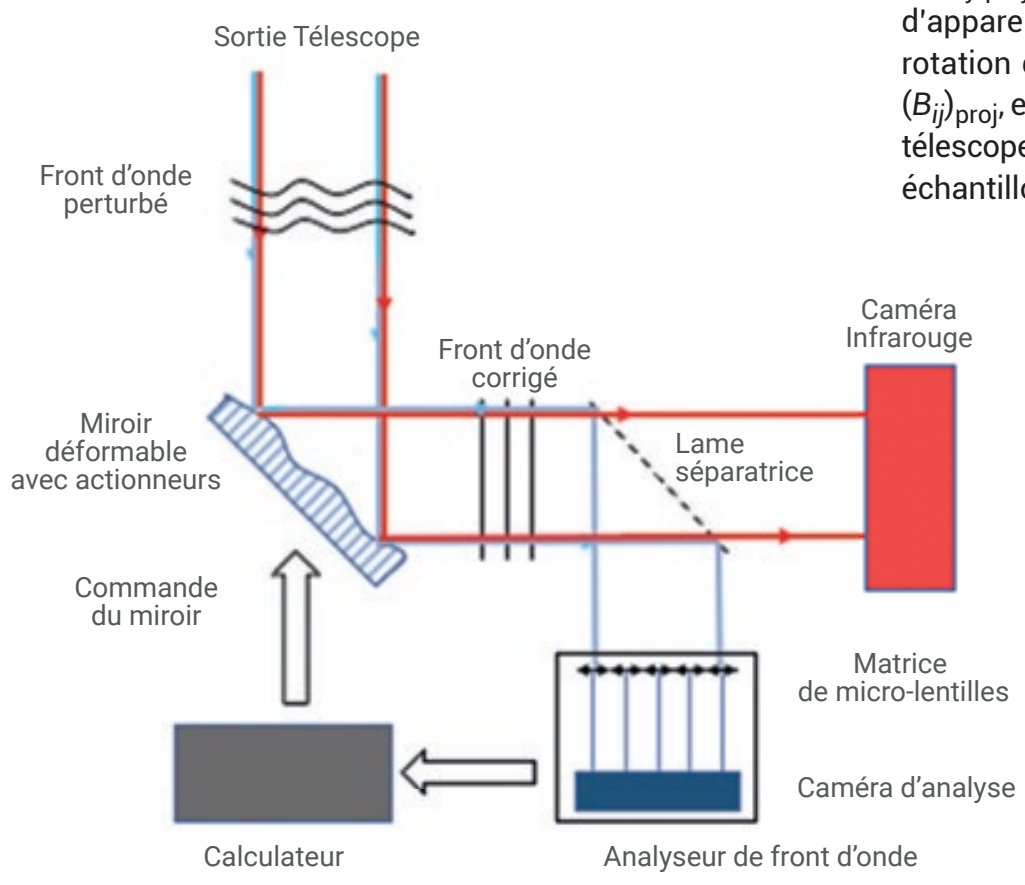

mesure la phase à la fréquence requise, fixée par l'état de l'atmosphère. Parfois, une étoile assez brillante est suffisamment proche de l'objet (dans le plan du ciel) pour être utilisée par l'analyseur comme référence et permettre la correction : on s'appuie alors sur la corrélation existante entre les perturbations affectant les parcours de deux ondes aux trajets très voisins. Sinon, la solution récemment adoptée consiste à exciter avec un laser, tiré par le télescope (voir la figure p. 21), la couche d'atomes de sodium fluorescents présents dans la haute stratosphère pour y créer une étoile artificielle, proche de la ligne de visée et servant de référence à l'analyseur.

L'interférométrie stellaire est une version développée de l'expérience des trous d'Young. Elle recherche une résolution angulaire plus élevée encore que $\lambda / D$, en combinant en un foyer unique $F$ la lumière issue de plusieurs télescopes $T_{1}, T_{2}, \ldots, T_{n}$, répartis sur un plan à des distances mutuelles $B_{i j}$ (bases interférométriques) et observant simultanément l'objet (fig. 1, p. 24). Si l'on veille à ce que les chemins optiques $\mathrm{T}_{\mathrm{i}} \mathrm{F}$ soient maintenus égaux à quelques nanomètres près, des franges d'Young se produisent en $F$, correspondant aux $n(n-1) / 2$ séparations entre télescopes. En échantillonnant ainsi le plan des fréquences spatiales, la mesure de l'amplitude et de la phase relative (nombre complexe) de ces systèmes de franges fournit des valeurs discrètes de la transformée de Fourier de l'image recherchée. En référence à l'onde incidente, c'est la valeur projetée de la base sur le plan d'onde qui définit la fréquence spatiale explorée, laquelle dépend donc de la direction de l'objet étudié. La fréquence la plus élevée est $\left(B_{i j}\right)_{\text {proj }} / \lambda$, quand $\left(B_{i j}\right)_{\text {proj }}$ est la plus grande valeur des séparations projetées entre télescopes, valeur qui peut atteindre plusieurs dizaines de fois leur diamètre ou davantage. La résolution atteinte est $\lambda /\left(B_{i j}\right)_{\text {proj }}$, et la qualité de l'image ainsi construite (fonction d'appareil) est d'autant meilleure que $n$ est grand. À $n$ fixé, la rotation de la Terre change au cours du temps la valeur des $\left(B_{i j}\right)_{\text {proj, }}$ et il est également possible de modifier les bases si les télescopes sont mobiles. L'une et l'autre conduisent à un meilleur chantillonnage du plan de Fourier, donc à une meilleure image.

E1. Principe de l'optique adaptative. Le schéma met en évidence les trois éléments essentiels d'une optique adaptative : l'analyseur de front d'onde, le calculateur (ordinateur), le miroir déformable, dont la surface est contrôlée par un ensemble d'actionneurs (piezoélectriques ou autres). L'analyseur échantillonne, à une longueur d'onde choisie (traits bleus) le front d'onde perturbé, réfléchi par une lame séparatrice, à l'aide d'une matrice de microlentilles, dont les images sur la caméra d'analyse fournissent la pente locale du front. Initialement, le front d'onde n'est pas corrigé, tandis que la figure présente la situation lorsque la boucle de rétroaction (flèches) fonctionne sur un front corrigé, qui est également reçu (traits rouges) par la caméra infrarouge, laquelle fournit alors l'image finale du champ étudié.

(Source : G. Rousset \& J.C. Fontanella). 


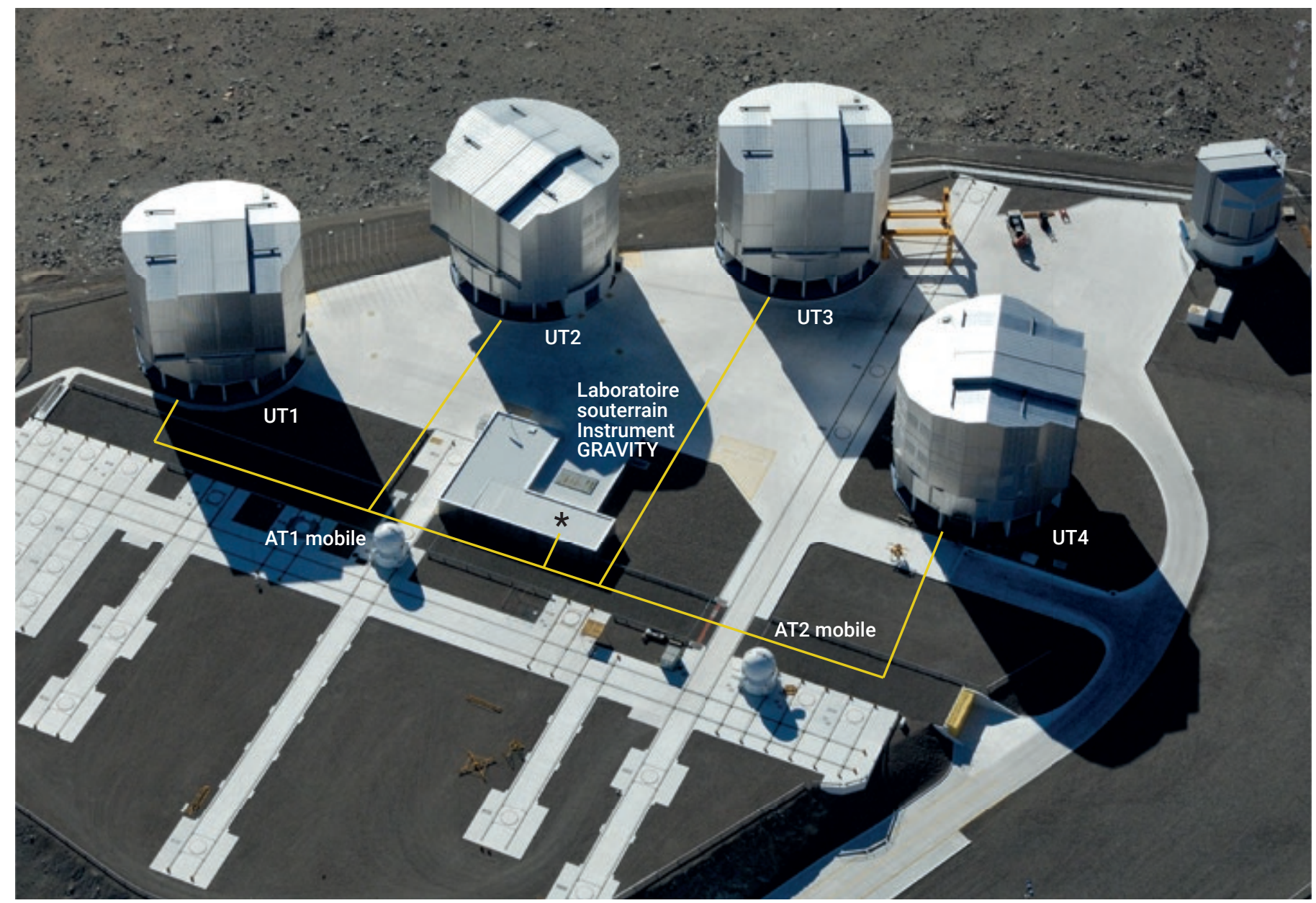

1. Le Very Large Telescope Interferometer (VLTI) sur le Cerro Paranal dans le désert d'Atacama (Chili). Le mode interférométrique du VLT consiste à coupler de façon cohérente soit les quatre télescopes (UT) de 8,2 m, soit les quatre télescopes auxiliaires (AT) de 1,8 m, mobiles sur rail, soit même une combinaison des UT et AT. Les différents faisceaux cheminent sous terre (lignes jaunes), après avoir reçu une première correction d'optique adaptative à leur sortie du télescope. Ils sont remis en phase par des lignes à retard variable, compensant notamment la rotation de la Terre, situées dans le laboratoire souterrain (marqué *), où ils sont finalement recombinés pour former la figure d'interférence, puis détectés par l'instrument Gravity, également situé dans ce laboratoire. (Source : ESO).

$\gg>$

de celle-ci. En outre, sur ce même télescope, le spectrographe Sinfoni, également corrigé par l'optique adaptative, mesure l'effet Doppler-Fizeau de S2 et donne la valeur de la composante radiale de sa vitesse.

De surcroit en 2003, le suivi de la région centrale met en évidence de brusques sursauts d'intensité lumineuse (flares), localisés sur la position de Sgr A*, dont l'interprétation n'est pas immédiate, mais qui sont sans doute liés au comportement de la matière dans le voisinage immédiat du trou noir [5]. La contrainte sur la compacité de l'objet restant limitée par la résolution angulaire, cette découverte rend plus nécessaire encore son amélioration.

\section{L'optique adaptative rend possible l'interférométrie optique du VLT}

L'étape suivante consiste donc à utiliser le mode interférométrique du VLT, appelé Very Large Telescope Interferometer (VLTI). Ce mode combine de façon cohérente les quatre télescopes de 8,2 m-Unit Telescopes ou UT (fig. 1). On mesure ainsi les fréquences spatiales d'une image dont la résolution atteint $\lambda / \mathrm{B}_{\mathrm{p}}$ où $\mathrm{B}_{\mathrm{p}}$ est la projection, sur le front d'onde incident, de la plus grande des bases interférométriques disponibles, soit ici B = $130 \mathrm{~m}$ entre les télescopes UT1 et UT4. La rotation de la Terre permet de couvrir un ensemble de fréquences spatiales, améliorant l'image obtenue alors par synthèse d'ouverture (voir l'encadré p. 23).
Décidé en 1987, leVLTI aboutit en 2002 soit quatre ans après la première lumière du VLT. Deux de ses UT sont alors couplés, et l'ensemble de quatre UT le sera en 2010 avec le système de recombinaison Pionier, réalisé en optique intégrée à l'Institut de Planétologie et d'Astrophysique de Grenoble.

Néanmoins, un chemin important restait à parcourir avant de pouvoir observer le cœur du centre galactique avec leVLTI, car des gains en sensibilité et en précision angulaire, chacun d'un facteur 1000, demeuraient nécessaires. Dans ce but et dès 2005, un nouvel instrument focal, appelé "Gravity", est mis en chantier par les équipes du Max-Planck Institute für extraterrestriche Physik (Garching) et de l'Observatoire de Paris. La combinaison d'une maitrise de l'optique adaptative, avec 
analyse de surface d'onde dans l'infrarouge, et de l'interférométrie de précision associée à la nature de l'environnement de Sgr A joue alors un rôle décisif [6]. L'instrument Gravity, devenu opérationnel à partir de 2016, exploite une circonstance favorable et tout à fait particulière à l'environnement immédiat de Sgr $A^{\star}$. En effet, la présence d'étoiles de référence, très proches de l'objet central et suffisamment brillantes, permet de faire fonctionner les systèmes d'optique adaptative sans étoile laser (voir l'encadré) et de stabiliser les franges d'interférence pour augmenter le temps de pose d'un facteur 1000 par rapport aux instruments conventionnels (fig. 2 et fig. 3 ).

La mesure finale fait appel à trois asservissements simultanés : le premier pour corriger les effets de la turbulence dès la sortie des quatre télescopes UT du VLT (optique adaptative sur l'étoile de référence IRS7) ; le deuxième au foyer commun pour corriger les résidus de turbulence sur une source interne avant recombinaison des faisceaux ; le troisième enfin pour mesurer les systèmes de franges dans le minuscule champ interférométrique de 2" $\times 2$ ", en asservissant sur l'étoile IRS16C (fig. 2 et fig. 3).

En amont de Gravity, des lignes à retard compensent les écarts de phase entre les faisceaux issus des quatre télescopes UT. Ces écarts sont provoqués par la rotation terrestre et la géométrie des bases par rapport à la direction de la source observée. Gravity forme et mesure à chaque instant six systèmes de franges en combinant les quatre faisceaux duVLTI par paires. L'image finale sera déduite de ces mesures (voir l'encadré). Des fibres monomodes en verre fluoré filtrent les turbulences résiduelles, compensent les écarts de différence de marche entre l'objet étudié et l'étoile de référence, et enfin contrôlent les polarisations. Ces techniques sont nées d'un long parcours sur des interféromètres équipés de télescopes de petit diamètre, tels que Fluor avec V. Coudé du Foresto ou 'Ohana avec G. Perrin. L'optique intégrée fait de Gravity un instrument compact et très stable, qui non seulement atteint dans l'image la limite de diffraction $\lambda / \mathrm{B}=2$ mas (millisecondes d'arc), mais peut la dépasser d'un facteur cent en faisant une mesure différentielle de distance entre les images de deux objets voisins, chacune pourtant limitée en résolution à $\lambda / \mathrm{B}$ (astrométrie interférométrique différentielle).

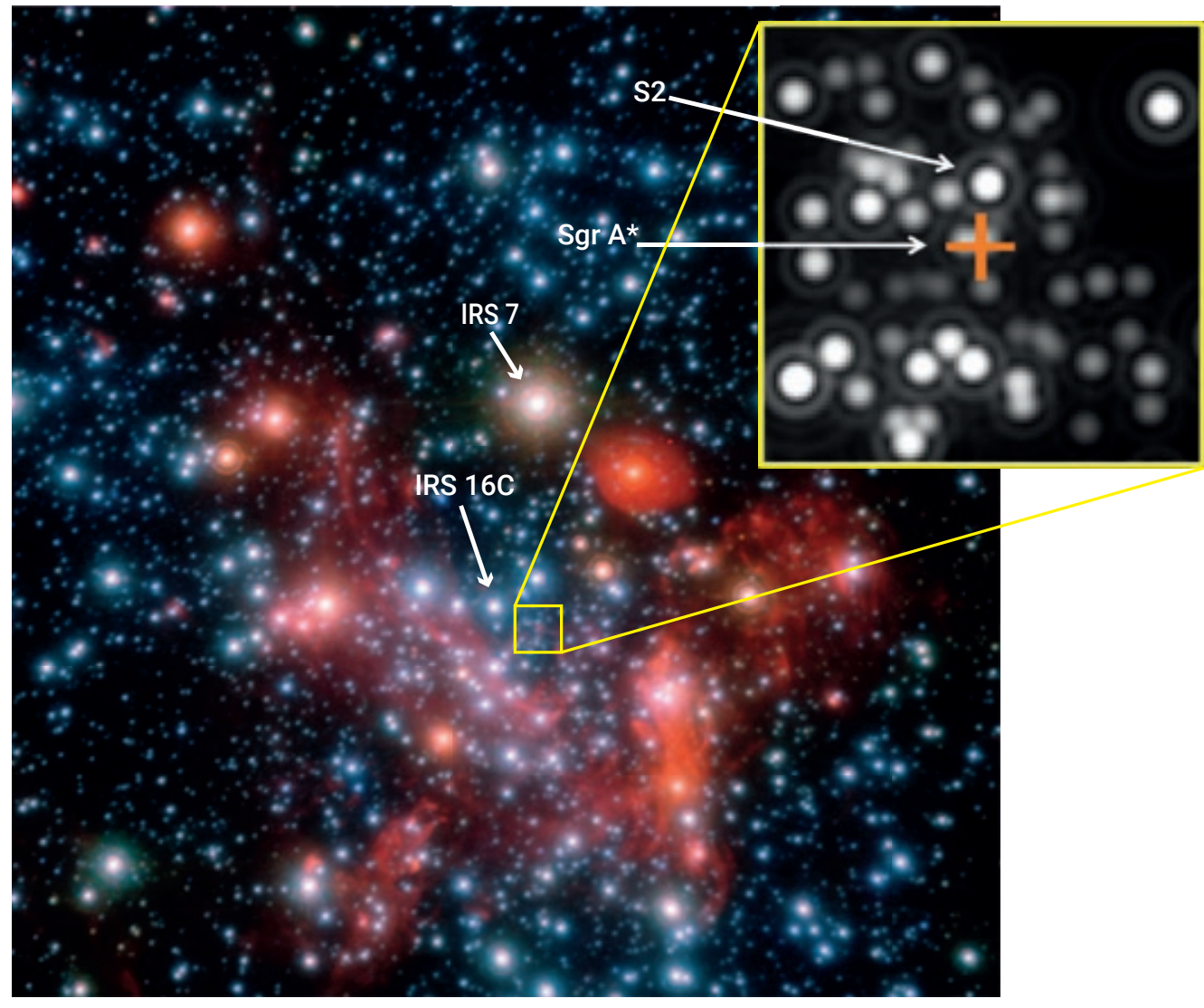

2. Images, dans le proche infrarouge, de la région centrale de la Voie lactée. Champ de 30 " x 30 " (secondes d'arc), avec insert de 1,4" x 1,4". L'étoile IRS7 est située à 6" de Sgr A* et sert de référence pour la correction adaptative. L'étoile IRS16C, située à 1" de Sgr $A^{*}$, se trouve dans le champ de vue de l'interféromètre (2" x 2"), non reporté sur la figure. Dans ce petit champ, le calcul comme les mesures montrent que les écarts de phase aléatoires, produits par des parcours atmosphériques relatifs à des objets distincts, demeurent fortement corrélés : on peut alors utiliser les franges sur IRS16C pour compenser ces écarts par un asservissement et faire des intégrations longues de plusieurs minutes, " en aveugle " sur celles de Sgr $A^{*}$. Les positions de Sgr A* et de l'étoile S2, alors non loin de son apocentre (point de l'orbite elliptique le plus éloigné du foyer), sont indiquées dans l'insert. Source : ESO.

Entre 2016 et 2020, Gravity a apporté des résultats majeurs. Le passage de l'étoile S2 au péricentre, en mai 2018, a été observé. Non seulement une meilleure détermination de la masse du trou noir a été obtenue par la caractérisation plus précise de l'ellipse parcourue, mais des effets relativistes en champ intense ont été mesurés : effet Doppler relativiste [7] et précession relativiste de la trajectoire elliptique [8]. Enfin, les brefs et intenses sursauts d'émission infrarouge ont été localisés avec précision dans le voisinage immédiat de Sgr A^. L'observation astrométrique différentielle de leur déplacement autour de Sgr A*, à la précision de 30 uas (microsecondes d'arc), permit de localiser leur orbite non loin de la dernière orbite circulaire stable (ISCO), à une distance de la singularité inférieure à quatre rayons de Schwarzschild (10 $\mu$ as) [9]. Il en résulte que la masse de 4,26 ( $\pm 0,1) 10^{6} \mathrm{M}_{\text {Soleil }}$ est contenue dans un volume proche de celui de l'horizon des événements (fig. 4).

Ces résultats exceptionnels n'épuisent pas le potentiel de la configuration adaptative et interférométrique de Gravity. Une évolution de ce dernier (Gravity+) reçoit la priorité pour une installation au Cerro Paranal en 2024-2025, avec des performances considérablement accrues. L'un des objectifs est de trouver une ou plusieurs étoiles, moins brillantes que S2, qui s'approcheraient davantage encore de Sgr A ${ }^{\star}$ et dont le mouvement permettrait un sondage de champs gravitationnels plus intenses encore. L'ambition est alors de mesurer la vitesse de rotation du trou noir, seul para- 


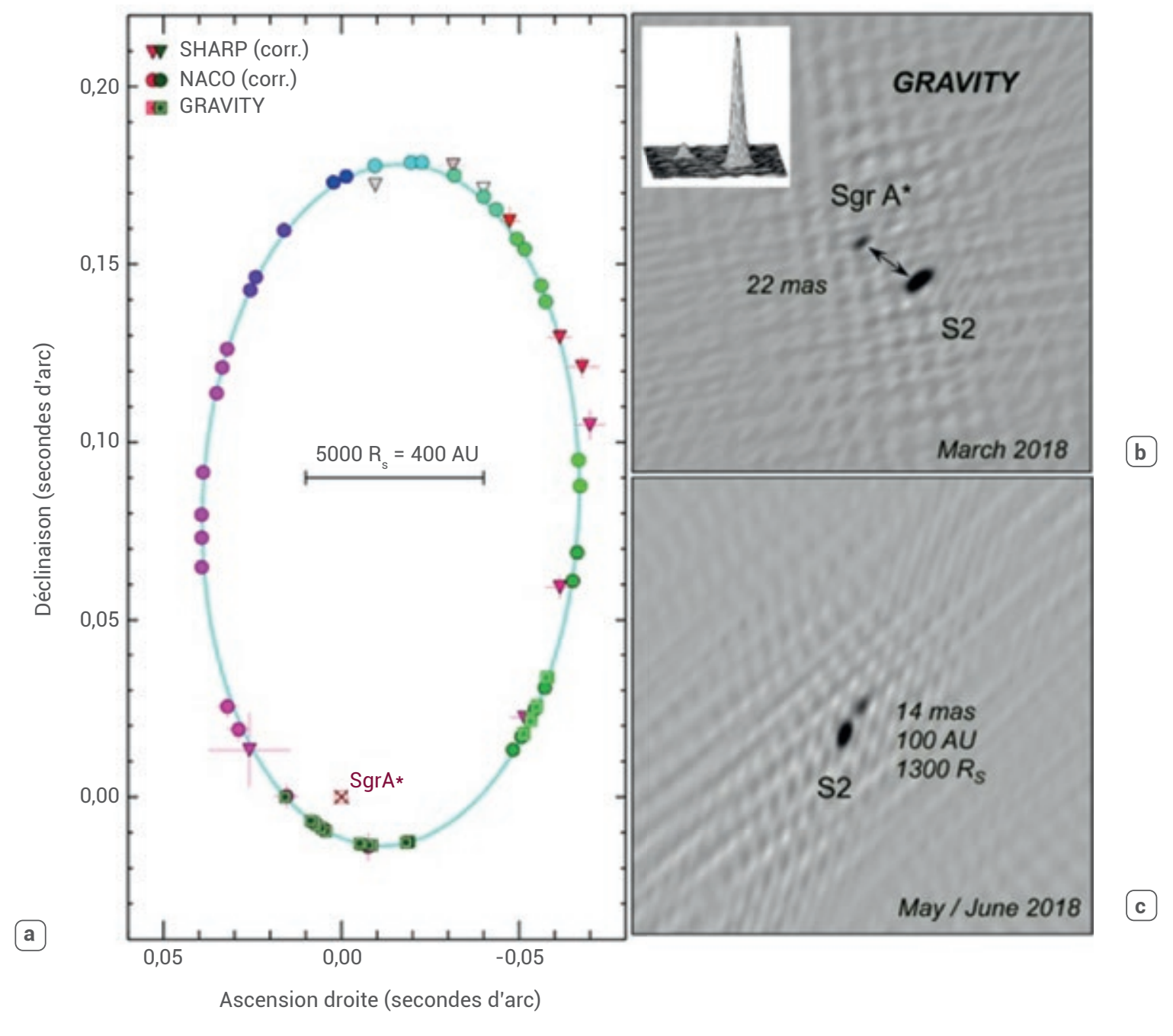

3. Orbite et images de l'étoile S2 [7]. (a) Orbite de S2 mesurée durant deux décennies par interférométrie des tavelures (caméra SHARP sur le télescope NTT de l'ESO, de diamètre 3,6 m), optique adaptative (instrument NACO sur le VLT, de diamètre 8,2 m) et interférométrie longue base (Gravity).

(b, c) Images de S2 prises juste avant et au moment du passage au péricentre en mai 2018. Le gain en résolution angulaire permet de clairement séparer S2 du trou noir. L'allongement apparent des objets traduit la fonction d'appareil de l'interféromètre (réponse impulsionnelle).

Source : GRAVITY Collaboration, A\&A 615 (2018) L15, reproduit avec la permission de l'ESO.

sensibilité permettant l'étude de ce trou noir central.

L'interféromètre intercontinental (programme Event Horizon Telescope EHT), réalisé par un ensemble de radiotélescopes millimétriques répartis sur la surface de la Terre, pôle Sud compris, possède une capacité d'imagerie supérieure à celle du VLTI, soit une résolution de $20 \mu$ as à $\lambda=1,3 \mathrm{~mm}$. L'observation par l'EHT du trou noir supermassif central dans la galaxie M87 a été publiée en 2019, mais celle de SgrA^ ne l'est pas encore [10].

\section{Conclusion}

L'argumentaire [1] du prix Nobel de physique 2020 met en lumière les apports théoriques de Roger Penrose à la relativité générale, ainsi que les résultats observationnels de Reinhard Genzel en Europe et d'Andrea Ghez aux États-Unis, obtenus par l'exploitation tenace des techniques de haute résolution angulaire pendant plusieurs décennies. Comme brièvement montré dans le présent texte, ces travaux ont utilisé les étapes successives d'une "lutte contre le flou " pour vaincre les limites de la
Pour des motifs essentiellement financiers, cette configuration a été mise en sommeil par la NASA à partir de 2008, si bien que le optique, combinant la résolution et la 


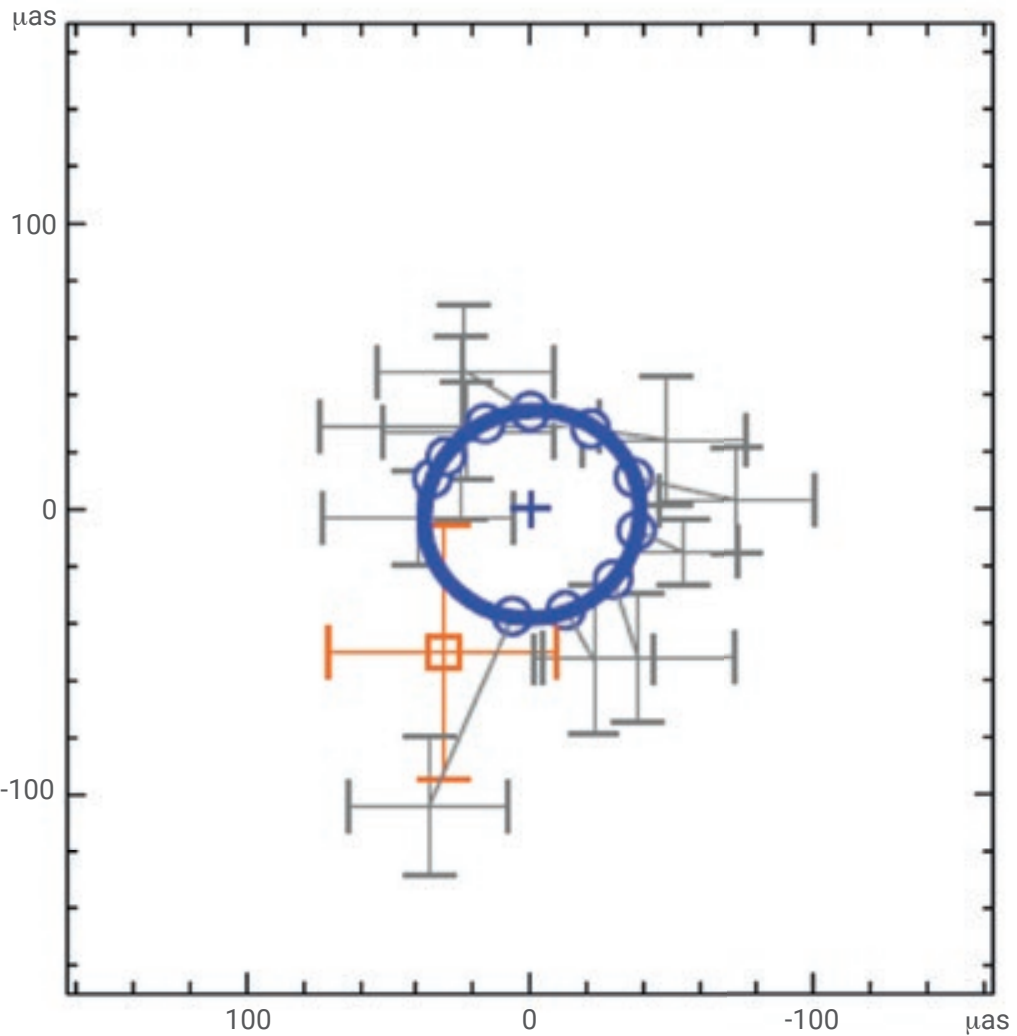

4. Mesures dans le proche infrarouge (croix grises) et modèle (en bleu) de la position apparente de Sgr $A^{*}$ lors d'un sursaut lumineux d'une trentaine de minutes (évènement du 22 juillet 2018) [9]. La croix orange marque la position du centre de masse déduite des mesures orbitales de l'étoile S2. Les axes sont en microsecondes d'arc ( $\mu$ as). Les incertitudes de position sont d'environ 30 uas, soit un centième de la limite de diffraction interférométrique pour une base de $130 \mathrm{~m}$ entre les deux télescopes du VLT les plus éloignés. L'instrument Gravity obtient cette précision par astrométrie différentielle entre deux faisceaux : I'un positionné sur Sgr A*, I'autre sur l'étoile IRS16C, distante d'une seconde d'arc. L'orbite relativiste calculée (en bleu) possède 1,17 fois le rayon de la dernière orbite circulaire stable d'un trou noir de Schwarzschild de 4 millions de masses solaires. Elle est la trace du mouvement du plasma porté à très haute température, potentiellement par reconnexion magnétique, autour de Sgr $A^{*}$. Il s'agit de la contrainte la plus forte sur la taille physique de Sgr $A^{*}$. L'écart entre la croix rouge et le centre de l'orbite bleue est probablement dû à l'imprécision de mesure ou à un écart entre la position apparente de Sgr $\mathrm{A}^{*}$ pendant un sursaut et sa position au repos, la base du jet dominant alors.

Source : GRAVITY Collaboration, A\&A 618 (2018) L10, reproduit avec la permission de l'ESO.

résolution des images astronomiques aux longueurs d'onde optiques, tout d'abord imposées par la turbulence atmosphérique présente même dans les meilleurs sites terrestres d'observation, puis par la diffraction sur des télescopes utilisés individuellement, enfin par la combinaison interférométrique sur de longues bases de plusieurs télescopes géants. Entre la limite imposée par le seeing, soit environ une seconde d'arc, et la fonction d'appareil de l'interféromètre VLTI, un gain en résolution de près d'un facteur mille est obtenu. Avec l'astrométrie interférométrique diffé- rentielle, un nouveau gain d'un facteur de l'ordre de 100 permet d'atteindre des précisions relatives de positionnement de l'ordre de la trentaine de microsecondes d'arc. Nul doute que ces méthodes nouvelles ne bénéficient à une foule d'autres problèmes, telle l'étude d'exoplanètes situées dans l'environnement immédiat de leur étoile, comme montré tout récemment par le VLTI et l'instrument Gravity, qui se verra doté dès 2024 d'améliorations considérables dans le cadre du projet Gravity+.
Références

1 The Nobel Committee for Physics, "Theoretical foundation for black holes and the supermassive object at the Galactic centre" (2020). www.nobelprize.org/uploads/2020/10/ advanced-physicsprize2020.pdf

2• A. Labeyrie, "Attainment of Diffraction Limited Resolution in Large Telescopes by Fourier Analysing Speckle Patterns in Star Images", A\&A 6 (1970) 85-87.

3. G. Rousset et al., "First diffractionlimited astronomical images with adaptative optics", A\&A 230 (1990) L29-L32.

4. A.M. Ghez et al., "High proper-motion stars in the vicinity of Sagittarius $A *$ : Evidence for a supermassive black hole at the center of our galaxy", Astrophys. J. 509 (1998) 678-686.

5• R. Genzel et al., "Near-infrared flares from accreting gas around the supermassive black hole at the Galactic Centre", Nature 425 (2003) 934-937.

6• F. Eisenhauer, G. Perrin et al., "Gravity: Observing the Universe in Motion", The Messenger, 143 (2011) 16-24.

7• GRAVITY Collaboration, R. Abuter et al., "Detection of the gravitational redshift in the orbit of the star S2 near the Galactic centre massive black hole", A\&A 615 (2018) L15.

8- GRAVITY Collaboration, R. Abuter et al., "Detection of the Schwarzschild precession in the orbit of the star S2 near the Galactic centre massive black hole", A\&A 636 (2020) L5.

9- GRAVITY Collaboration, R. Abuter et al., "Detection of orbital motions near the last stable circular orbit of the massive black hole SgrA*", A\&A 618 (2018) L10.

10 F. Combes, «Première image d'un trou noir ", Reflets de la physique 64 (2020) 23-25.

11· D. Rouan, « L'E-ELT, un œil géant pour scruter l'univers », Reflets de la physique 47-48 (2016) 78-83. 